\title{
INVOLUTIONS WHOSE FIXED SET HAS THREE OR FOUR COMPONENTS: A SMALL CODIMENSION PHENOMENON
}

\author{
E. M. BARBARESCO, P. E. DESIDERI and P. L. Q. PERGHER*
}

\begin{abstract}
Let $T: M \rightarrow M$ be a smooth involution on a closed smooth manifold and $F=\bigcup_{j=0}^{n} F^{j}$ the fixed point set of $T$, where $F^{j}$ denotes the union of those components of $F$ having dimension $j$ and thus $n$ is the dimension of the component of $F$ of largest dimension. In this paper we prove the following result, which characterizes a small codimension phenomenon: suppose that $n \geq 4$ is even and $F$ has one of the following forms: 1) $F=F^{n} \cup F^{3} \cup F^{2} \cup$ \{point ; 2) $F=F^{n} \cup F^{3} \cup F^{2}$; 3) $F=F^{n} \cup F^{3} \cup$ \{point\}; or 4) $F=F^{n} \cup F^{3}$. Also, suppose that the normal bundles of $F^{n}, F^{3}$ and $F^{2}$ in $M$ do not bound. If $k$ denote the codimension of $F^{n}$, then $k \leq 4$. Further, we construct involutions showing that this bound is best possible in the cases 2) and 4 ), and in the cases 1) and 3) when $n$ is of the form $n=4 t$, with $t \geq 1$.
\end{abstract}

\section{Introduction}

Throughout this paper, the involved cobordism notions will be understood in the unoriented sense. Let $F$ be a disjoint (finite) union of smooth and closed manifolds and $M$ be a smooth and closed manifold equipped with a smooth involution $T: M \rightarrow M$ whose fixed point set is $F$. Suppose that $F$ is not a boundary. If $n$ is the dimension of a component of $F$ of maximal dimension and $k$ is the codimension of this component, then $k \leq \frac{3}{2} n$; this follows from the famous Five Halves Theorem of J. Boardman, announced in [1], and its strengthened version of [11]. In fact, the Five Halves Theorem asserts that this is valid when $M$ is not a boundary, and in [11] R. E. Stong and C. Kosniowski established the same conclusion under the weaker hypothesis that $(M, T)$ is a nonbounding involution. The assertion then follows from the fact that the equivariant cobordism class of $(M, T)$ is determined by the cobordism class of the normal bundle of $F$ in $M$ (see [4]).

The generality of this result, which is valid for every $n \geq 1$, allows the possibility that fixed components of all dimensions $j, 0 \leq j \leq n$, occur; in this way, it is natural to ask whether there exist better bounds for $k$ when we

\footnotetext{
* The authors were partially supported by CNPq, FAPESP and CAPES.

Received 18 November 2010, in final form 10 May 2011.
} 
omit some components of $F$ and restrict the set of involved dimensions $n$. This question is inspired by the following results of the literature:

1) (R. E. Stong and C. Kosniowski, [11], 1978): if $F=F^{n}$ has constant dimension $n$ (and so $F^{n}$ is not a boundary), then $k \leq n$. For each fixed $n$, with the exception of the dimensions $n=1$ and $n=3$, the maximal value $k=n$ is achieved by taking the involution $\left(F^{n} \times F^{n}, T\right)$, where $F^{n}$ is any nonbounding $n$-dimensional manifold and $T$ is the twist involution, $T(x, y)=(y, x)$; that is, one has in this case an (best possible) improvement for the Boardman bound by omitting the $j$-dimensional components of $F$ with $j<n$ and excluding $n=1$ and 3 .

2) (D. C. Royster, [15], 1980): in this case, the result in question is referring to an intriguing improvement for the Boardman bound, which characterizes a small codimension phenomenon, given by $n$ odd and the omission of the $j$-dimensional components of $F$ with $0<j<n$. Specifically, if $n$ is odd and $F$ has the form $F=F^{n} \cup\{$ point $\}$, then $k \leq 1$. Evidently, this bound is best possible, and is realized by the involution $\left(R P^{n+1}, T\right)$, where $R P^{n+1}$ is the $(n+1)$-dimensional real projective space and $T\left[x_{0}, x_{1}, \ldots, x_{n+1}\right]=$ $\left[-x_{0}, x_{1}, \ldots, x_{n+1}\right]$, with $n$ odd.

This class of problems was introduced by P. Pergher in [12], where the above Royster result was enlarged in the following way: if $F$ has the form $F=F^{n} \cup\{$ point $\}$, where $n=2 p$ with $p$ odd, then $k \leq p+3$. This case $\left(F=F^{n} \cup\{\right.$ point $\}$ ) was completed by R. Stong and P. Pergher in [14], in the following (best possible) way: writing $n=2^{p} q$, where $p \geq 0$ and $q$ is odd, then $k \leq n+p-q+1$ if $p \leq q$ and $k \leq n+2^{p-q}$ if $p>q$.

With the cases $F=F^{n}$ and $F=F^{n} \cup$ \{point completed, the next natural step is the case $F=F^{n} \cup F^{j}, 0<j<n$. Concerning this more general case, recently some advances have been obtained; specifically, we find best possible bounds for $F=F^{n} \cup F^{1}$ in [9] and [10], $F=F^{n} \cup F^{2}$ in [5], [6] and [7], $F=F^{n} \cup F^{n-1}$ in [8] and $F=F^{n} \cup F^{j}$ with $F^{j}$ indecomposable in [13]. Among these results, one particularly finds other cases involving small codimension phenomena: if $F=F^{n} \cup F^{1}$ and $n$ is even, then $k \leq 2$ [9], and if $F=F^{n} \cup F^{2}$ and $n$ is odd, then $k \leq 3$ [6]. Further, these bounds are best possible. For $F=F^{n}, F=F^{n} \cup F^{n-1}, F=F^{n} \cup F^{1}$ ( $n$ odd) and $F=F^{n} \cup F^{2}$ ( $n$ even), small codimensions do not occur, which means that $k$ is not limited as a function of $n$.

If the fixed point set $F$ of an involution $(M, T)$ is expressed as $F=$ $\bigcup_{j=0}^{n} F^{j}$, where $F^{j}$ denotes the union of those components of $F$ having dimension $j$, then in all the discussion above each $F^{j}$ can be supposed connected. This follows from the fact that, given two $k$-dimensional vector bundles over closed $j$-dimensional manifolds, $\xi \rightarrow V$ and $v \rightarrow N$, the connected sum of $\xi$ and $v, \xi \sharp v$ (which is a $k$-dimensional vector bundle over $V \sharp N$ ), is cobordant as 
a bundle to the disjoint union $\xi \cup v$. From [4], we then conclude that $(M, T)$ is equivariantly cobordant to an involution whose $j$-dimensional part of the fixed point set is connected. So the cases $F=F^{n}$ and $F=F^{n} \cup F^{j}, 0 \leq j<n$, can be referred to as the one component case and two components case, respectively. Further, if the normal bundle of some $F^{j}$ in $M$ is a boundary, it can be equivariantly removed to give a new involution, equivariantly cobordant to $(M, T)$ and with fixed point set $F-F^{j}$ (see [4]). Therefore such $F^{j}$ have no influence in the context of looking for bounds for the possible codimensions. This leads us to assume throughout this paper, without mention, that the normal bundle over each mentioned $F^{j}$ does not bound.

The goal of this paper is to start the study of small codimension phenomena when the number of components of $F$ is greater than 2 . While in the one and two components cases one has standard sources of examples, the difficulty with more components lies in constructing examples to detect the sharpness of the obtained bounds. Also, the characteristic number computations to find bounds require more sophistication.

We will prove the following

THEOREM 1. Let $(M, T)$ be an involution having fixed point set of the form $F=F^{n} \cup P$, where $n \geq 4$ is even and $P$ has the possible forms:

1) $P=F^{3} \cup F^{2} \cup\{$ point $\}$;

2) $P=F^{3} \cup F^{2}$;

3) $P=F^{3} \cup\{$ point $\}$;

4) $P=F^{3}$.

As previously mentioned, suppose that the normal bundle to each $F^{j}$ does not bound. Then, if $k$ is the codimension of $F^{n}, k \leq 4$. Further, there are involutions showing that this bound is best possible in the cases 2) and 4), and in the cases 1) and 3) with $n$ of the form $n=4 t, t \geq 1$.

Section 2 will be devoted to the construction of the above mentioned maximal involutions. The main tool will be a combination of a construction of P. Conner and E. Floyd of [4] with a result of R. E. Stong and P. Pergher of [14]. In Section 3 we prove the part " $k \leq 4$ ", using the Conner and Floyd theory and some special polynomials in the characteristic classes of total spaces of projective space bundles, introduced by Stong and Pergher in [14].

\section{Maximal involutions}

In order to construct the examples, we need to give some preliminaries and to establish some notations.

If $(M, T)$ is an involution pair with fixed point set $F$ and $\eta \rightarrow F$ is the normal bundle of $F$ in $M$, we call $\eta \rightarrow F$ the fixed data of $(M, T)$. 
For a vector bundle $\eta \rightarrow F$ and a natural number $p \geq 1$, write $p \eta \rightarrow F$ for the Whitney sum of $p$ copies of $\eta ; R \rightarrow F$ will denote the one dimensional trivial vector bundle. If $\eta$ is $k$-dimensional, write $W(\eta)=1+w_{1}(\eta)+w_{2}(\eta)+$ $\cdots+w_{k}(\eta) \in H^{*}\left(F, Z_{2}\right)$ for the Stiefel Whitney class of $\eta$; if $F$ is a closed smooth $n$-dimensional manifold, $W(F)=1+w_{1}(F)+w_{2}(F)+\cdots+w_{n}(F)$ will denote the Stiefel Whitney class of the tangent bundle of $F$.

From [4], one has an algebraic scheme to determine the cobordism class of $\eta$, given by the set of Whitney numbers (or characteristic numbers) of $\eta$; such modulo 2 numbers are obtained by evaluating $n$-dimensional $Z_{2}$-cohomology classes of the form $w_{i_{1}}(F) w_{i_{2}}(F) \ldots w_{i_{r}}(F) w_{j_{1}}(\eta) w_{j_{2}}(\eta) \ldots w_{j_{s}}(\eta) \in$ $H^{n}\left(F, Z_{2}\right)$ (that is, with $i_{1}+i_{2}+\cdots+i_{r}+j_{1}+j_{2}+\cdots+j_{s}=n$ ) on the fundamental homology class $[F] \in H_{n}\left(F, Z_{2}\right)$.

The following construction of P. Conner and E. Floyd will be useful (see [4]): let $\left(M^{n}, T\right)$ be an involution defined on a closed $n$-dimensional manifold $M^{n}$ with fixed data $\eta \rightarrow F$. On $S^{1} \times M^{n}$, consider the involutions $-\mathrm{Id} \times T$ and $c \times \mathrm{Id}$, where $S^{1}$ is the unit circle in the complex numbers, Id is the identity map and $c$ is the complex conjugation. Note that $-\mathrm{Id} \times T$ is free and commutes with $c \times \mathrm{Id}$, hence $c \times \mathrm{Id}$ induces an involution on the orbit space $S^{1} \times M^{n} /-\mathrm{Id} \times T$, which is a closed $(n+1)$-dimensional manifold. This involution, denoted by $\Gamma\left(M^{n}, T\right)$, has $(R \oplus \eta \rightarrow F) \cup\left(R \rightarrow M^{n}\right)$ as fixed data. If $M^{n}$ bounds, $R \rightarrow M^{n}$ bounds as a line bundle, so $\Gamma\left(M^{n}, T\right)$ is equivariantly cobordant to an involution with fixed data $R \oplus \eta \rightarrow F$. If $S^{1} \times M^{n} /-\mathrm{Id} \times T$ is a boundary, we can repeat the process taking $\Gamma^{2}\left(M^{n}, T\right)$, and so on.

We are now ready to give the required examples. For $n \geq 1$, denote by $\lambda_{n} \rightarrow R P^{n}$ the canonical line bundle over the $n$-dimensional real projective space, and for $n \geq 0$ and $p \geq 0$, consider the involution $\left(R P^{n+p+1}, T_{n, p}\right)$ defined in homogeneous coordinates by

$$
T_{n, p}\left[x_{0}, x_{1}, \ldots, x_{n+p+1}\right]=\left[-x_{0},-x_{1}, \ldots,-x_{n}, x_{n+1}, \ldots, x_{n+p+1}\right] .
$$

The fixed data of $T_{n, p}$ is

$$
\left((p+1) \lambda_{n} \rightarrow R P^{n}\right) \cup\left((n+1) \lambda_{p} \rightarrow R P^{p}\right) .
$$

Now take $n \geq 4$ even. Since $n+3$ is odd, $R P^{n+3}$ bounds, and thus $\Gamma\left(R P^{n+3}, T_{n, 2}\right)$ is equivariantly cobordant to an involution $\left(B^{n+4}, f\right)$ with fixed data

$$
\left(3 \lambda_{n} \oplus R \rightarrow R P^{n}\right) \cup\left((n+1) \lambda_{2} \oplus R \rightarrow R P^{2}\right) ;
$$

also, the fixed data of $\left(R P^{n+4}, T_{n, 3}\right)$ is

$$
\left(4 \lambda_{n} \rightarrow R P^{n}\right) \cup\left((n+1) \lambda_{3} \rightarrow R P^{3}\right) .
$$


Set $\alpha \in H^{1}\left(R P^{n}, Z_{2}\right)$ and $\beta \in H^{1}\left(R P^{3}, Z_{2}\right)$ for the generators of the onedimensional $Z_{2}$-cohomology groups. Note that $4 \lambda_{n}, 3 \lambda_{n} \oplus R$ and $(n+1) \lambda_{2} \oplus R$ do not bound because $R P^{n}$ and $R P^{2}$ do not bound; also, $(n+1) \lambda_{3}$ and $4 \lambda_{n} \cup\left(3 \lambda_{n} \oplus R\right)$ do not bound because

$$
\left(w_{1}\left((n+1) \lambda_{3}\right)\right)^{3}\left[R P^{3}\right]=((n+1) \beta)^{3}\left[R P^{3}\right]=\beta^{3}\left[R P^{3}\right]=1
$$

and

$$
\begin{aligned}
& \left(w_{1}\left(4 \lambda_{n} \cup\left(3 \lambda_{n} \oplus R\right)\right)\right)^{n}\left[R P^{n} \cup R P^{n}\right] \\
& \quad=\left(w_{1}\left(4 \lambda_{n}\right)\right)^{n}\left[R P^{n}\right]+\left(w_{1}\left(3 \lambda_{n} \oplus R\right)\right)^{n}\left[R P^{n}\right] \\
& \quad=(4 \alpha)^{n}\left[R P^{n}\right]+(3 \alpha)^{n}\left[R P^{n}\right]=0+\alpha^{n}\left[R P^{n}\right]=1 .
\end{aligned}
$$

Thus $\left(R P^{n+4}, T_{n, 3}\right)$ and $\left(R P^{n+4}, T_{n, 3}\right) \cup\left(B^{n+4}, f\right)$ are involutions showing that the bound $k \leq 4$ is best possible in the cases 4 ) and 2).

The examples for the cases 1) and 3) are a little more subtle. Write $n=2^{d} q$, where $d \geq 1$ and $q$ is odd. In [14], R. Stong and P. Pergher showed that the underlying manifold of $\Gamma^{j}\left(R P^{n+1}, T_{n, 0}\right)$ bounds for $j=1$ if $d=1$, and for $j \leq 2^{d}-2$ if $d>1$. If $n$ is of the form $n=4 t, t \geq 1$, then $d \geq 2$, and thus the underlying manifold of $\Gamma^{2}\left(R P^{n+1}, T_{n, 0}\right)$ bounds. It follows that $\Gamma^{3}\left(R P^{n+1}, T_{n, 0}\right)$ is equivariantly cobordant to an involution $\left(W^{n+4}, g\right)$ with fixed data

$$
\left(\lambda_{n} \oplus 3 R \rightarrow R P^{n}\right) \cup((n+4) R \rightarrow\{\text { point }\}) .
$$

Now $\lambda_{n} \oplus 3 R$ and $(n+4) R \rightarrow$ ppoint $\}$ do not bound, and $4 \lambda_{n} \cup\left(\lambda_{n} \oplus 3 R\right)$ does not bound because

$$
\left(w_{1}\left(4 \lambda_{n} \cup\left(\lambda_{n} \oplus 3 R\right)\right)\right)^{n}\left[R P^{n} \cup R P^{n}\right]=\alpha^{n}\left[R P^{n}\right]=1 .
$$

Thus the involution $\left(R P^{n+4}, T_{n, 3}\right) \cup\left(W^{n+4}, g\right)$ realizes the bound $k \leq 4$ in the case 3).

Finally, note that $4 \lambda_{n} \cup\left(3 \lambda_{n} \oplus R\right) \cup\left(\lambda_{n} \oplus 3 R\right)$ does not bound because the base space $R P^{n} \cup R P^{n} \cup R P^{n}$ is cobordant to $R P^{n}$. Then this vector bundle is cobordant to a nonbounding 4-dimensional vector bundle $\mu \rightarrow G^{n}$, where $G^{n}$ is a connected closed $n$-dimensional manifold. From [4], there exists an involution $\left(K^{n+4}, h\right)$ equivariantly cobordant to $\left(R P^{n+4}, T_{n, 3}\right) \cup\left(B^{n+4}, f\right) \cup$ $\left(W^{n+4}, g\right)$, and with fixed data

$$
\begin{aligned}
& \left(\mu \rightarrow G^{n}\right) \cup\left((n+1) \lambda_{3} \rightarrow R P^{3}\right) \\
& \qquad\left((n+1) \lambda_{2} \oplus R \rightarrow R P^{2}\right) \cup((n+4) R \rightarrow\{\text { point }\}) .
\end{aligned}
$$

Then $\left(K^{n+4}, h\right)$ realizes the bound in question in the case 1$)$. 


\section{A small codimension phenomenom}

Let $(M, T)$ be an involution having fixed point set of the form $F=F^{n} \cup P$, where $n \geq 4$ is even and $P$ is as described in the statement of the main theorem. We will prove that, if $k$ is the codimension of $F^{n}$, then $k \leq 4$.

If $\eta \rightarrow F$ is the fixed data of an involution, denote by $E$ the total space of the real projective space bundle associated to $\eta, R P(\eta) \rightarrow F$, and by $\xi \rightarrow E$ the line bundle of the double cover $S(\eta) \rightarrow E, S(\eta)$ the sphere bundle of $\eta$. It is known, from the Conner and Floyd exact sequence of [4], that $\xi \rightarrow E$ bounds as a line bundle. Then, if $\operatorname{dim}(E)=r$, any class $P=P\left(w_{1}(E), \ldots, w_{r}(E), w_{1}(\xi)\right) \in H^{r}\left(E, Z_{2}\right)$, given by a polynomial of dimension $r$ in the classes $w_{i}(E)$ and $w_{1}(\xi)$, gives the zero characteristic number $P[E]$; in this case, $P[E]$ splits into a modulo 2 sum of factors corresponding to the connected components of $F$.

Returning to our particular case, denote by $E_{i}, i=0,2,3$ and $n$, the total space of the projective space bundle corresponding to the $i$-dimensional component, and by $\xi_{i} \rightarrow E_{i}$ the corresponding line bundle. Our strategy will be first to select four special polynomials, $P_{1}, P_{2}, P_{3}$ and $P_{4}$, with dimension $n+k-1$, leading to a modulo 2 system of equations

$$
\left\{\begin{array}{l}
\sum_{i=1}^{4} P_{1}\left[E_{i}\right]=0 \\
\sum_{i=1}^{4} P_{2}\left[E_{i}\right]=0 \\
\sum_{i=1}^{4} P_{3}\left[E_{i}\right]=0 \\
\sum_{i=1}^{4} P_{4}\left[E_{i}\right]=0
\end{array}\right.
$$

$P_{1}, P_{2}, P_{3}$ and $P_{4}$ will be built under the hypothesis, by contradiction, that $k>4$. After computation of the terms $P_{i}\left[E_{j}\right]$, this system will be reduced to a system of equations in four variables, these variables being four special characteristic numbers of the normal bundle over the 3 -dimensional component. The zero solution will be the unique solution of this system. Then we will prove that these four characteristic numbers determine the cobordism class of any bundle over a 3-dimensional closed manifold. This will imply that the normal bundle over the 3-dimensional component bounds, giving the contradiction.

Denote by $\eta_{i} \rightarrow F^{i}, i=0,2,3$ and $n$, the normal bundle over the $i$ dimensional component. To avoid excessive notation, write $W\left(F^{i}\right)=1+$ 
$w_{1}+w_{2}+\cdots+w_{i}, W\left(\eta_{i}\right)=1+v_{1}+\cdots+v_{n+k-i}$ and $W\left(\xi_{i}\right)=1+c$ for the Stiefel-Whitney classes.

From [2, p. 517], the Stiefel-Whitney class of $E_{i}$ is

$$
W\left(E_{i}\right)=\left(1+w_{1}+\cdots+w_{i}\right)\left((1+c)^{n+k-i}+(1+c)^{n+k-i-1} v_{1}+\cdots+v_{n+k-i}\right),
$$

where here we are suppressing bundle maps.

To construct the polynomials $P_{i}, i=1,2,3$ and 4 , we consider the class

$$
\widetilde{W}\left(E_{i}\right)=\frac{W\left(E_{i}\right)}{(1+c)^{k}}=\frac{1+w_{1}\left(E_{i}\right)+w_{2}\left(E_{i}\right)+\cdots}{(1+c)^{k}}=1+\widetilde{W}_{1}+\widetilde{W}_{2}+\cdots
$$

This kind of class was introduced by Stong and Pergher in [14]. Each $\widetilde{W}_{j}$ is a polynomial in the classes $w_{s}\left(E_{i}\right)$ and $c$, which means that it can be used to yield the required polynomials. Thus, since $k>4$, it makes sense to set $P_{1}=$ $\widetilde{W}_{1}^{n+4} c^{k-5}, P_{2}=\widetilde{W}_{1}^{n} \widetilde{W}_{2}^{2} c^{k-5}$ and $P_{3}=\widetilde{W}_{1}^{n-1} \widetilde{W}_{2} \widetilde{W}_{3} c^{k-5}$. We recall that, if Sq is the Steenrod operation, then the Wu formula implies that $\mathrm{Sq}^{j}$ evaluated on a characteristic class of a bundle gives a polynomial in the characteristic classes of this bundle. Therefore, by the Cartan formula, $\operatorname{Sq}^{1}\left(\widetilde{W}_{3}\right)$ is a polynomial in the classes $w_{s}\left(E_{i}\right)$ and $c$; we define $P_{4}=\widetilde{W}_{1}^{n} \mathrm{Sq}^{1}\left(\widetilde{W}_{3}\right) c^{k-5}$. Next we compute $P_{i}\left[E_{j}\right]$ for $i=1,2,3,4$ and $j=0,2,3, n$.

1) Since $F^{0}=\{$ point $\}, E_{0}=R P^{n+k-1}$ and

$$
\widetilde{W}\left(E_{0}\right)=\frac{(1+c)^{n+k}}{(1+c)^{k}}=(1+c)^{n} .
$$

Because $n$ is even, $\widetilde{W}_{1}=n c=0$. Thus $P_{i}=0$ for $i=1,2,3,4$.

2) On $F^{2}$, one has

$$
\widetilde{W}\left(E_{2}\right)=\left(1+w_{1}+w_{2}\right)\left[(1+c)^{n-2}+(1+c)^{n-3} v_{1}+(1+c)^{n-4} v_{2}\right] .
$$

Because $n$ is even,

$$
\widetilde{W}_{1}=(n-2) c+v_{1}+w_{1}=v_{1}+w_{1} .
$$

Since $\operatorname{dim}\left(F^{2}\right)=2$ and $n \geq 4$, by dimensional reasons $P_{i}=0$ for $i=$ $1,2,3,4$.

3) On $F^{n}$, one has

$$
\begin{aligned}
\widetilde{W}\left(E_{n}\right)=\left(1+w_{1}+\cdots+w_{n}\right) & {\left[1+\frac{v_{1}}{1+c}\right.} \\
+ & \left.\frac{v_{2}}{1+c^{2}}+\frac{v_{3}}{(1+c)^{3}}+\cdots+\frac{v_{k}}{(1+c)^{k}}\right] .
\end{aligned}
$$


Then,

$$
\begin{aligned}
& \widetilde{W}_{1}=w_{1}+v_{1}, \\
& \widetilde{W}_{2}=v_{1} c+v_{2}+w_{1} v_{1}+w_{2}, \\
\text { and } \quad \widetilde{W}_{3} & =v_{1} c^{2}+w_{1} v_{1} c+v_{3}+w_{1} v_{2}+w_{2} v_{1}+w_{3} .
\end{aligned}
$$

Since $\operatorname{Sq}^{1}\left(c^{2}\right)=0$, the Cartan formula gives

$$
\mathrm{Sq}^{1}\left(v_{1} c^{2}+w_{1} v_{1} c\right)=v_{1}^{2} c^{2}+w_{1} v_{1} c^{2}+w_{1} v_{1}^{2} c+w_{1}^{2} v_{1} c
$$

and so $\widetilde{W}_{1}^{n} \mathrm{Sq}^{1}\left(\widetilde{W}_{3}\right)=0$ by dimensional reasons. Also

$$
\begin{aligned}
\widetilde{W}_{1}^{n+4} & =\left(w_{1}+v_{1}\right)^{n+4}, \\
\widetilde{W}_{1}^{n} \widetilde{W}_{2}^{2} & =\left(w_{1}+v_{1}\right)^{n}\left(v_{1}^{2} c^{2}+v_{2}^{2}+w_{1}^{2} v_{1}^{2}+w_{2}^{2}\right)
\end{aligned}
$$

and $\quad \widetilde{W}_{1}^{n-1} \widetilde{W}_{2} \widetilde{W}_{3}=\left(w_{1}+v_{1}\right)^{n-1}\left(v_{1} c+v_{2}+w_{1} v_{1}+w_{2}\right)$

$$
\cdot\left(v_{1} c^{2}+w_{1} v_{1} c+v_{3}+w_{1} v_{2}+w_{2} v_{1}+w_{3}\right) .
$$

Note that each term of the above polynomials has a factor of dimension at least $n+1$ coming from the cohomology of $F^{n}$, which gives $P_{i}=0$ for $i=1,2,3,4$.

4) On $F^{3}$, one has

$$
\begin{aligned}
\widetilde{W}\left(E_{3}\right)=\left(1+w_{1}+w_{2}+w_{3}\right)\left[(1+c)^{n-3}\right. \\
\left.+(1+c)^{n-4} v_{1}+(1+c)^{n-5} v_{2}+(1+c)^{n-6} v_{3}\right] .
\end{aligned}
$$

Because $n$ is even and by dimensional reasons, $\widetilde{W}_{1}=(n-3) c+v_{1}+w_{1}=$ $c+v_{1}+w_{1}$ and

$$
\begin{aligned}
\widetilde{W}_{1}^{n+4} & =\left(v_{1}+w_{1}+c\right)^{n+4}=\sum_{j=0}^{n+4}\left(\begin{array}{c}
n+4 \\
j
\end{array}\right)\left(v_{1}+w_{1}\right)^{j} c^{n+4-j} \\
& = \begin{cases}c^{n+4}, & \text { if } n \equiv 0 \bmod 4, \\
c^{n+4}+\left(v_{1}^{2}+w_{1}^{2}\right) c^{n+2}, & \text { if } n \equiv 2 \bmod 4 .\end{cases}
\end{aligned}
$$

Thus

$$
P_{1}\left[E_{3}\right]= \begin{cases}c^{n+k-1}\left[E_{3}\right], & \text { if } n \equiv 0 \bmod 4, \\ \left(c^{n+k-1}+\left(v_{1}^{2}+w_{1}^{2}\right) c^{n+k-3}\right)\left[E_{3}\right], & \text { if } n \equiv 2 \bmod 4 .\end{cases}
$$


Denoting by

$$
\bar{W}\left(\eta_{3}\right)=\frac{1}{W\left(\eta_{3}\right)}=1+\bar{v}_{1}+\bar{v}_{2}+\bar{v}_{3}
$$

the dual Stiefel-Whitney class of $\eta_{3}$, the Conner formula of [3] gives that

$$
x c^{n+k-i}\left[E_{3}\right]=x \bar{v}_{4-i}\left[F^{3}\right],
$$

for every $x \in H^{i-1}\left(F^{3}\right)$ and $1 \leq i \leq 4$ (this follows from the fact that $H^{*}\left(E_{3}, Z_{2}\right)$ is a free $H^{*}\left(F^{3}, Z_{2}\right)$-module with basis $\left\{1, c, c^{2}, \ldots, c^{n+k-4}\right\}$, with multiplication determined by the relation $c^{n+k-3}=v_{1} c^{n+k-4}+v_{2} c^{n+k-5}+$ $\left.v_{3} c^{n+k-6}\right)$. Since $\bar{v}_{1}=v_{1}$ and $\bar{v}_{3}=v_{1}^{3}+v_{3}$, this gives

$$
P_{1}\left[E_{3}\right]= \begin{cases}\bar{v}_{3}\left[F^{3}\right]=\left(v_{1}^{3}+v_{3}\right)\left[F_{3}\right], & \text { if } n \equiv 0 \bmod 4, \\ \left(\bar{v}_{3}+\left(v_{1}^{2}+w_{1}^{2}\right) \bar{v}_{1}\right)\left[F^{3}\right]=\left(v_{3}+w_{1}^{2} v_{1}\right)\left[F^{3}\right], & \text { if } n \equiv 2 \bmod 4 .\end{cases}
$$

To compute $P_{i}\left[E_{3}\right], i=2,3$ and 4 , we use the same approach above and the following ingredients:

i) the relations $w_{3}=w_{1} w_{2}=w_{1}^{3}=0$, which follow from the fact that every 3 -dimensional manifold bounds; and the relations $w_{2} v_{1}=w_{1}^{2} v_{1}, w_{1} v_{1}^{2}=0$ and $w_{1} v_{2}=v_{1} v_{2}+v_{3}$, which are valid for every vector bundle over a 3dimensional manifold (see the proof of the fact stated at the end of the paper).

ii)

$$
\begin{aligned}
\widetilde{W}_{2} & =\left(\begin{array}{c}
n-3 \\
2
\end{array}\right) c^{2}+(n-4) v_{1} c+(n-3) w_{1} c+v_{2}+w_{1} v_{1}+w_{2} \\
& = \begin{cases}w_{1} c+v_{2}+w_{1} v_{1}+w_{2}, & \text { if } n \equiv 0 \bmod 4, \\
c^{2}+w_{1} c+v_{2}+w_{1} v_{1}+w_{2}, & \text { if } n \equiv 2 \bmod 4 ;\end{cases}
\end{aligned}
$$

iii)

$$
\begin{array}{r}
\tilde{W}_{3}=\left(\begin{array}{c}
n-3 \\
3
\end{array}\right) c^{3}+\left(\begin{array}{c}
n-4 \\
2
\end{array}\right) v_{1} c^{2}+\left(\begin{array}{c}
n-3 \\
2
\end{array}\right) w_{1} c^{2}+(n-5) v_{2} c \\
+(n-4) w_{1} v_{1} c+(n-3) w_{2} c+w_{2} v_{1}+w_{1} v_{2}+v_{3}
\end{array}
$$


iv)

$$
\operatorname{Sq}^{1}\left(\tilde{W}_{3}\right)^{\dagger}=\left\{\begin{aligned}
\left(v_{2}+w_{2}\right) c^{2}+\left(v_{1} v_{2}+v_{3}\right) c, & \text { if } n \equiv 0 \bmod 4 \\
c^{4}+\left(v_{1}^{2}+w_{1}^{2}+v_{2}+w_{2}\right) c^{2} & \\
+\left(v_{1} v_{2}+v_{3}\right) c, & \text { if } n \equiv 2 \bmod 4
\end{aligned}\right.
$$

v)

$$
\begin{aligned}
\widetilde{W}_{1}^{n} & =\sum_{j=0}^{n}\left(\begin{array}{l}
n \\
j
\end{array}\right)\left(v_{1}+w_{1}\right)^{j} c^{n-j}=c^{n}+\left(\begin{array}{l}
n \\
2
\end{array}\right)\left(v_{1}^{2}+w_{1}^{2}\right) c^{n-2} \\
& = \begin{cases}c^{n}, & \text { if } n \equiv 0 \bmod 4, \\
c^{n}+\left(v_{1}^{2}+w_{1}^{2}\right) c^{n-2}, & \text { if } n \equiv 2 \bmod 4,\end{cases}
\end{aligned}
$$

and

$$
\begin{aligned}
\widetilde{W}_{1}^{n-1}= & c^{n-1}+\left(v_{1}+w_{1}\right) c^{n-2} \\
& +\left(\begin{array}{c}
n-1 \\
2
\end{array}\right)\left(v_{1}^{2}+w_{1}^{2}\right) c^{n-3}+\left(\begin{array}{c}
n-1 \\
3
\end{array}\right)\left(v_{1}+w_{1}\right)^{3} c^{n-4} \\
= & \left\{\begin{array}{rr}
c^{n-1}+\left(v_{1}+w_{1}\right) c^{n-2} \\
+\left(v_{1}^{2}+w_{1}^{2}\right) c^{n-3}+\left(v_{1}+w_{1}\right)^{3} c^{n-4}, & \text { if } n \equiv 0 \bmod 4, \\
c^{n-1}, & \text { if } n \equiv 2 \bmod 4 .
\end{array}\right.
\end{aligned}
$$

With these data in hand we obtain, by doing a routine calculation, that

$$
\begin{aligned}
& P_{2}\left[E_{3}\right]= \begin{cases}w_{1}^{2} v_{1}\left[F^{3}\right], & \text { if } n \equiv 0 \bmod 4, \\
v_{3}\left[F^{3}\right], & \text { if } n \equiv 2 \bmod 4,\end{cases} \\
& P_{3}\left[E_{3}\right]= \begin{cases}w_{1} v_{2}\left[F^{3}\right], & \text { if } n \equiv 0 \bmod 4, \\
\left(v_{3}+w_{1}^{2} v_{1}+w_{1} v_{2}\right)\left[F^{3}\right], & \text { if } n \equiv 2 \bmod 4,\end{cases}
\end{aligned}
$$

and

$$
P_{4}\left[E_{3}\right]= \begin{cases}\left(w_{1}^{2} v_{1}+v_{3}\right)\left[F^{3}\right], & \text { if } n \equiv 0 \bmod 4, \\ \left(v_{1}^{3}+w_{1}^{2} v_{1}\right)\left[F^{3}\right], & \text { if } n \equiv 2 \bmod 4 .\end{cases}
$$

Taking into account that a cohomology class $v \in H^{3}\left(F^{3}, Z_{2}\right)$ is zero if and only if $v\left[F^{3}\right]=0$, we conclude that our initial system is reduced to the

\footnotetext{
† In fact, by the $\mathrm{Wu}$ formula one has $\mathrm{Sq}^{1}\left(v_{2}+w_{2}\right)=v_{1} v_{2}+v_{3}+w_{1} w_{2}+w_{3}=v_{1} v_{2}+v_{3}$; also, $\mathrm{Sq}^{1}\left(w_{2} v_{1}+w_{1} v_{2}+v_{3}\right)=0$. The remaining calculation follows from the Cartan Formula.
} 
following systems of equations in the variables $v_{1}^{3}, v_{3}, w_{1} v_{2}$ and $w_{1}^{2} v_{1}$ :

$$
\left\{\begin{aligned}
v_{1}^{3}+v_{3} & =0 \\
w_{1}^{2} v_{1} & =0 \\
w_{1} v_{2} & =0 \\
w_{1}^{2} v_{1}+v_{3} & =0
\end{aligned} \quad \text { if } \quad n \equiv 0 \bmod 4\right.
$$

and

$$
\left\{\begin{aligned}
v_{3}+w_{1}^{2} v_{1} & =0 \\
v_{3} & =0 \\
v_{3}+w_{1}^{2} v_{1}+w_{1} v_{2} & =0 \\
v_{1}^{3}+w_{1}^{2} v_{1} & =0
\end{aligned} \text { if } n \equiv 2 \bmod 4 .\right.
$$

In both cases, $v_{1}^{3}=v_{3}=v_{2} w_{1}=w_{1}^{2} v_{1}=0$ is the unique solution. Thus, the following fact will end our task:

FACT. The cobordism class of any vector bundle over a 3-dimensional manifold $F^{3}$ is determined by the Whitney numbers $v_{1}^{3}\left[F^{3}\right], v_{3}\left[F^{3}\right], v_{2} w_{1}\left[F^{3}\right]$ and $v_{1} w_{1}^{2}\left[F^{3}\right]$.

Proof. The complete list of Whitney numbers in this case is $w_{3}\left[F^{3}\right]$, $w_{1} w_{2}\left[F^{3}\right], w_{1}^{3}\left[F^{3}\right], v_{3}\left[F^{3}\right], v_{1} v_{2}\left[F^{3}\right], v_{1}^{3}\left[F^{3}\right], w_{1} v_{1}^{2}\left[F^{3}\right], w_{1} v_{2}\left[F^{3}\right], w_{1}^{2} v_{1}\left[F^{3}\right]$ and $w_{2} v_{1}\left[F^{3}\right]$.

The first reduction is given by the fact that $F^{3}$ bounds: $w_{3}\left[F^{3}\right]=$ $w_{1} w_{2}\left[F^{3}\right]=w_{1}^{3}\left[F^{3}\right]=0$.

Now let $U=1+u_{1}$ be the Wu class of $F^{3}$. Then

$$
W\left(F^{3}\right)=1+w_{1}+w_{2}+w_{3}=\operatorname{Sq}(U)=1+u_{1}+u_{1}^{2},
$$

which gives $w_{2}=u_{1}^{2}=w_{1}^{2}$ and the next reduction, $w_{2} v_{1}=w_{1}^{2} v_{1}$.

If $x \in H^{2}\left(F^{3}, Z_{2}\right)$, it is known that $\mathrm{Sq}^{1}(x)=u_{1} x$; also, $\mathrm{Sq}^{1}\left(v_{1}^{2}\right)=0$. Then one has the reduction $w_{1} v_{1}^{2}=u_{1} v_{1}^{2}=\mathrm{Sq}^{1}\left(v_{1}^{2}\right)=0$.

Finally, by the $\mathrm{Wu}$ formula,

$$
w_{1} v_{2}=u_{1} v_{2}=\operatorname{Sq}^{1}\left(v_{2}\right)=v_{1} v_{2}+v_{3},
$$

which makes $v_{1} v_{2}\left[F^{3}\right]$ unnecessary.

ACKNOWLEDGEMENT. We would like to express our gratitude to the referee for suggestions that helped to clarify considerably the original version; we also thank Bjørn Ian Dundas for the guidance concerning the referee suggestions. 


\section{REFERENCES}

1. Boardman, J. M., On manifolds with involution, Bull. Amer. Math. Soc. 73 (1967), 136-138.

2. Borel, A., and Hirzebruch, F., Characteristic classes and homogeneous spaces I, Amer. J. Math. 80 (1958), 458-538.

3. Conner, P. E., The bordism class of a bundle space, Michigan Math. J. 14 (1967), 289-303.

4. Conner, P. E., and Floyd, E. E., Differentiable Periodic Maps, Ergebn. Math. Grenzgeb. (NF) 33, Springer, Berlin 1964.

5. Figueira, F. G., and Pergher, P. L. Q., Bounds on the dimension of manifolds with involution fixing $F^{n} \cup F^{2}$, Glasgow Math. J. 50 (2008), 595-604.

6. Figueira, F. G., and Pergher, P. L. Q., Dimensions of fixed point sets of involutions, Arch. Math. (Basel) 87 (2006), 280-288.

7. Figueira, F. G., and Pergher, P. L. Q., Involutions fixing $F^{n} \cup F^{2}$, Topology Appl. 153 (2006), 2499-2507.

8. Figueira, F. G., and Pergher, P. L. Q., Two commuting involutions fixing $F^{n} \cup F^{n-1}$, Geom. Dedicata 117 (2006), 181-193.

9. Kelton, S. M., Involutions fixing $R P^{j} \cup F^{n}$, Topology Appl. 142 (2004), 197-203.

10. Kelton, S. M., Involutions fixing $R P^{j} \cup F^{n} I I$, Topology Appl. 149 (2005), 217-226.

11. Kosniowski, C., and Stong, R. E., Involutions and characteristic numbers, Topology 17 (1978), 309-330.

12. Pergher, P. L. Q., Bounds on the dimension of manifolds with certain $Z_{2}$ fixed sets, Mat. Contemp. 13 (1996), 269-275.

13. Pergher, P. L. Q., Involutions fixing $F^{n} \cup\{$ Indecomposable $\}$, Canadian Math. Bull. 55 (2012), 164-171.

14. Pergher, P. L. Q., and Stong, R. E., Involutions fixing $\{$ point $\} \cup F^{n}$, Transform. Groups 6 (2001), 79-86.

15. Royster, D. C., Involutions fixing the disjoint union of two projective spaces, Indiana Univ. Math. J. 29 (1980), 267-276.

DEPARTAMENTO DE MATEMÁTICA

UNIVERSIDADE FEDERAL DE SÃO CARLOS

CAIXA POSTAL 676

SÃO CARLOS, SP 13565-905 BRAZIL

E-mail: evelinmbarbaresco@dm.ufscar.br patricia.desideri@gmail.com pergher@dm.ufscar.br 